\title{
Superposition Operators on Dirichlet Spaces *
}

by

\author{
Patrick J. Fitzsimmons
}

\begin{abstract}
In the context of a strongly local Dirichlet space we show that if a function mapping the real line to itself (and fixing the origin) operates by composition on the left to map the Dirichlet space into itself, then the function is necessarily locally Lipschitz continuous. If, in addition, the Dirichlet space contains unbounded elements, then the function must be globally Lipschitz continuous. The proofs rely on a co-area formula for condenser potentials.
\end{abstract}

\section{Introduction}

Let $(\mathcal{E}, \mathcal{D})$ be a strongly local regular Dirichlet form as in [13]. Specifically, let $E$ be a locally compact second countable metric space, and $m$ a Radon measure on $E$ with full support. The domain $\mathcal{D}$ of the Dirichlet form $\mathcal{E}$ is a vector space contained and dense in $L^{2}(m)$. The symmetric bilinear form $\mathcal{E}: \mathcal{D} \times \mathcal{D} \rightarrow \mathbf{R}$ is non-negative definite, and the augmented form $\mathcal{E}_{1}:(u, v) \mapsto \mathcal{E}(u, v)+(u, v)_{m}$ is a Hilbertian inner product on $\mathcal{D}$. (Here $(u, v)_{m}:=\int_{E} u v d m$ is the inner product in $L^{2}(m)$; the associated norm is $\|u\|_{2}$.) The form $\mathcal{E}$ is closable in the sense that if $\left\{u_{n}\right\}$ is an $\mathcal{E}$-Cauchy sequence with $\lim _{n}\left\|u_{n}\right\|_{2}=0$, then $\lim _{n} \mathcal{E}\left(u_{n}, u_{n}\right)=0$ as well. Furthermore, $(\mathcal{E}, \mathcal{D})$ enjoys the following contraction property: If $K: \mathbf{R} \rightarrow \mathbf{R}$ satisfies the conditions

$$
K(0)=0
$$

and

$$
|K(t)-K(s)| \leq M|t-s| \quad \text { for all } s, t \in \mathbf{R},
$$

then

$$
K \circ u \in \mathcal{D} \quad \text { for all } u \in \mathcal{D} \text {, }
$$

* 2000 Mathematics Subject Classification: Primary 31C25, secondary 60J45, 46E35 
and

$$
\mathcal{E}(K \circ u, K \circ u) \leq M^{2} \cdot \mathcal{E}(u, u) \quad \text { for all } u \in \mathcal{D} \text {. }
$$

In the sequel we shall describe the property (1.3) by saying that " $K$ operates on $\mathcal{D}$ ". Notice that the elements of $\mathcal{D}$ are actually classes of functions equal a.e. with respect to $m$, but that the superposition operation $u \mapsto K \circ u$ preserves $m$-classes. The modifier "regular" indicates that $\mathcal{D} \cap C_{0}(E)$ is $\mathcal{E}_{1}$-dense in $\mathcal{D}$ and uniformly dense in $C_{0}(E)$. (Here, $C_{0}(E)$ denotes the class of real-valued continuous functions on $E$ with compact support.) Finally, "strongly local" means that whenever $u$ and $v$ are elements of $\mathcal{D}$ such that the measures $u \cdot m$ and $v \cdot m$ are of compact support and $v$ is constant on a neighborhood of the support of $u \cdot m$, then necessarily $\mathcal{E}(u, v)=0$.

Our goal in this paper is to examine the following question: If $K$ operates on $\mathcal{D}$, must it be Lipschitz continuous, at least locally? This problem has been posed and solved by Marcus and Mizel [17] in the context of Sobolev spaces $W^{1, p}(\Omega)(1 \leq p<\infty)$, where the bounded Euclidean domain $\Omega \subset \mathbf{R}^{d}$ is assumed to satisfy a cone condition. [The critical case $p=d \geq 2$, left open in [17], was treated by Bourdaud [5].] Their work was extended to general Euclidean domains of finite measure by Labutin [15]. [See [2] for a monograph treatment of these and related questions.] The intersection of our context with that of these authors is the case $p=2$, corresponding to Brownian motion. They found that if $K$ operates on $W^{1,2}(\Omega)$, then $K$ is locally Lipschitz (i.e., $K$ is absolutely continuous and $\left.K^{\prime} \in L_{\text {loc }}^{\infty}(\mathbf{R})\right)$; if, in addition, $W^{1,2}(\Omega)$ contains essentially unbounded elements, then $K^{\prime} \in L^{\infty}(\mathbf{R})$. We shall see that these two conclusions remain valid for superpositions operating on the Dirichlet space of a general diffusion process. (The second conclusion requires a mild secondary hypothesis in the general case.)

Our methods are quite different from those used in the papers cited above and the constructions involved are less intricate. This simplification is due to a systematic use of condenser potentials, and to a related variant of the co-area formula that is of independent interest. This formula, an explicit instance of the "energy image density" formula of Bouleau and Hirsch [4], was suggested by a calculation found in recent work of Fukushima and Ying $[\mathbf{1 4}]$.

Our arguments are largely probabilistic, and in the balance of this section we shall describe the probabilistic context in which we shall be working, and we shall review some 
relevant facts. The precise statement of our main result, and its proof, will occupy the next section.

Associated with the Dirichlet form $(\mathcal{E}, \mathcal{D})$ is a symmetric Markov process $X=\left(\Omega, \mathcal{F}, \mathcal{F}_{t}, \theta_{t}, X_{t}, \mathbf{P}^{x}\right)$, with symmetry measure $m$. The process $X$ is a Hunt process; in particular, the transition semigroup of $X$, defined by

$$
P_{t} f(x):=\mathbf{P}^{x}\left[f\left(X_{t}\right)\right], \quad t \geq 0,
$$

maps $b \mathcal{B}(E)$ (the class of bounded real-valued $\mathcal{B}(E)$-measurable functions on $E$ ) into itself. Here $\mathcal{B}(E)$ denotes the class of Borel subsets of $E$ and $\mathbf{P}^{x}[Z]$ denotes the expectation of the random variable $Z$ with respect to the measure $\mathbf{P}^{x}$. As noted already, the semigroup $\left(P_{t}\right)$ is $m$-symmetric:

$$
\left(f, P_{t} g\right)_{m}=\left(P_{t} f, g\right)_{m}, \quad f, g \in p \mathcal{B}(E) .
$$

Because $(\mathcal{E}, \mathcal{D})$ is strongly local, $X$ is a diffusion in the sense that

(1.7)(i) The $\mathbf{P}^{m}$-completion $\left(\mathcal{F}_{t}\right)_{t \geq 0}$ of the natural filtration $\sigma\left\{X_{s} ; 0 \leq s \leq t\right\}, t \geq 0$, is quasi-left-continuous and the lifetime of $X$, denoted $\zeta$, is an $\left(\mathcal{F}_{t}\right)$ predictable stopping time;

(1.7)(ii) $t \mapsto X_{t}$ is continuous on $\left[0, \zeta\left[, \mathbf{P}^{m}\right.\right.$-a.s.

[Here $\mathbf{P}^{m}[\cdot]:=\int_{E} \mathbf{P}^{x}[\cdot] m(d x)$. Also, $\zeta$, the lifetime of $X$, is the time at which $X$ retires to the cemetery state $\Delta$, which has been adjoined to $E$ (as the point at infinity if $E$ is not compact) to allow for the possible mass defect $P_{t} 1_{E}(x)<1$. By convention any function (resp. measure) defined on $E(\operatorname{resp} . \mathcal{E})$ is extended to the cemetery state $\Delta$ by declaring its value at $\Delta$ (resp. $\{\Delta\})$ to be 0.$]$ Consequently, every $\left(\mathcal{F}_{t}\right)$-stopping time is $\mathbf{P}^{m}$-predictable, and every $\left(\mathcal{F}_{t}\right)$-martingale has continuous paths $\left(\mathbf{P}^{m}\right.$-a.s. $)$. See $[\mathbf{2 1} ; \S 47]$. As general references on Markov processes, the reader can consult [3] and [21].

Let us now recall more about the specifics of the association between $(\mathcal{E}, \mathcal{D})$ and $X$. Because of $(1.6),\left(P_{t}\right)$ restricted to $b \mathcal{B}(E) \cap L^{2}(m)$ extends uniquely to a strongly continuous contraction semigroup of self-adjoint operators in $L^{2}(m)$. The Dirichlet form $\mathcal{E}$ is given by the formula

$$
\mathcal{E}(u, v):=\lim _{t \downarrow 0} t^{-1}\left(u, v-P_{t} v\right)_{m},
$$


and its domain is specified by

$$
\mathcal{D}:=\left\{u \in L^{2}(m) ; \sup _{t>0} t^{-1}\left(u, u-P_{t} u\right)_{m}<\infty\right\}
$$

We shall often write $\mathcal{E}(u)$ instead of $\mathcal{E}(u, u)$.

(1.10) Remark. In the discussion at hand, there is nothing to be gained by working in the more general context of quasi-regular Dirichlet forms, as developed in [16]. Indeed, the main result of [7] asserts that every quasi-regular Dirichlet form is quasi-homeomorphic to a regular Dirichlet form, and it is a simple matter to check that our hypotheses and our conclusions are preserved by quasi-homeomorphisms.

The hitting time $\inf \left\{t>0 ; X_{t} \in B\right\}$ of $B \in \mathcal{B}(E)$ is denoted by $T_{B}$; the exit time from $B$, namely $T_{B^{c}}$, is denoted by $\tau(B)$. An increasing sequence $\left\{B_{n}\right\}$ of Borel subsets of $E$ is a nest provided $\mathbf{P}^{m}\left[\lim _{n} \tau\left(B_{n}\right)<\zeta\right]=0$. The reader is referred to [16; Lemma IV.4.5] for a characterization of this notion in terms of the Dirichlet form $(\mathcal{E}, \mathcal{D})$.

Each element $u \in \mathcal{D}$ admits a Borel measurable $m$-modification $\tilde{u}$ such that $t \mapsto \tilde{u}\left(X_{t}\right)$ is right continuous on $\left[0, \infty\left[, \mathbf{P}^{m}\right.\right.$-a.s.; because $X$ is a diffusion, we then have the automatic left continuity of $t \mapsto \tilde{u}\left(X_{t}\right)$ on $] 0, \zeta\left[, \mathbf{P}^{m}\right.$-a.s. The function $\tilde{u}$ is quasi-continuous in the sense that there is a nest $\left(K_{n}\right)$ of compact subsets of $E$ such that $\left.\tilde{u}\right|_{K_{n}} \in C\left(K_{n}\right)$ for every $n$.

A Borel set $N \subset E$ is said to be exceptional provided $\mathbf{P}^{m}[\tau(E \backslash N)<\zeta]=0$. It can be shown that $N \in \mathcal{B}(E)$ is exceptional if and only if there is a nest of compacts $\left\{K_{n}\right\}$ such that $N \subset \bigcap_{n} K_{n}^{c}$. A statement or property depending on $x \in E$ is said to hold quasi-everywhere (q.e.) provided the set of points where it fails to hold is exceptional.

Given $u \in \mathcal{D}$, we have Fukushima's decomposition [13; Theorem 5.2.2]:

$$
\tilde{u}\left(X_{t}\right)-\tilde{u}\left(X_{0}\right)=M_{t}^{u}+N_{t}^{u}, \quad \text { for all } t \geq 0, \mathbf{P}^{x} \text {-a.s. for q.e. } x \in E,
$$

where $M^{u}$ and $N^{u}$ are continuous additive functionals (CAFs) of $X, M^{u}$ is a martingale such that $\sup _{t>0} t^{-1} \mathbf{P}^{m}\left[\left[M_{t}^{u}\right]^{2}\right]<\infty$, and $\lim _{t \rightarrow 0} t^{-1} \mathbf{P}^{m}\left[\left[N_{t}^{u}\right]^{2}\right]=0$. This decomposition is unique.

If $u \in \mathcal{D}$ then the martingale $\mathrm{CAF} M^{u}$ admits a quadratic variation process $\left\langle M^{u}\right\rangle$; i.e., $\left\langle M^{u}\right\rangle$ is a PCAF such that $\left(M^{u}\right)^{2}-\left\langle M^{u}\right\rangle$ is a martingale on $[0, \zeta[$. The Revuz measure 
of $\left\langle M^{u}\right\rangle$ (the so-called energy measure of $u$ ) is the smooth measure $\mu_{\langle u\rangle}$ on $E$ determined by

$$
\mu_{\langle u\rangle}(f)=\uparrow \lim _{t \rightarrow 0} t^{-1} \mathbf{P}^{m}\left[\int_{0}^{t} f\left(X_{s}\right) d\left\langle M^{u}\right\rangle_{s}\right], \quad f \in p \mathcal{B}(E) .
$$

The measure $\mu_{\langle u\rangle}$ has finite total mass; indeed,

$$
\mathcal{E}(u, u)=\frac{1}{2} \mu_{\langle u\rangle}(E), \quad u \in \mathcal{D} \text {. }
$$

Given two elements $u$ and $v$ of $\mathcal{D}$, the quadratic covariation $\left\langle M^{u}, M^{v}\right\rangle$ is a (signed) CAF of $X$ and, since $\left\langle M^{u}, M^{v}\right\rangle=(1 / 4)\left[\left\langle M^{u+v}\right\rangle-\left\langle M^{u-v}\right\rangle\right]$, we have

$$
\mu_{\langle u, v\rangle}=\frac{1}{4}\left[\mu_{\langle u+v\rangle}-\mu_{\langle u-v\rangle}\right]
$$

and

$$
\mathcal{E}(u, v)=\frac{1}{2} \mu_{\langle u, v\rangle}(E), \quad u, v \in \mathcal{D} \text {. }
$$

We close this section with a result that will be useful in some of the constructions of the next section.

(1.15) Proposition. Let $F: \mathbf{R} \rightarrow \mathbf{R}$ be an absolutely continuous function with $F(0)=0$. Given $u \in \mathcal{D}$, if $F \circ u \in L^{2}(m)$ and $F^{\prime} \circ \tilde{u} \in L^{2}\left(\mu_{\langle u\rangle}\right)$, then $F \circ u \in \mathcal{D}$ and

$$
d \mu_{\langle F \circ u\rangle}=\left[F^{\prime} \circ \tilde{u}\right]^{2} d \mu_{\langle u\rangle} .
$$

Proof. Let $\xi$ denote the image of $(1 / 2) \mu_{\langle u\rangle}$ under the mapping $x \mapsto \tilde{u}(x)$. This is a finite measure on the Borel subsets of $\mathbf{R}$. Moreover, $\xi$ is absolutely continuous with respect to Lebesgue measure on $\mathbf{R}$ by [4; Theorem I.7.1.1]. Thus the derivative $F^{\prime} \in L_{\text {loc }}^{1}(\mathbf{R})$ is uniquely determined up to $\xi$-null sets, and the hypothesis $F^{\prime} \circ \tilde{u} \in L^{2}\left(\mu_{\langle u\rangle}\right)$ (equivalently $\left.F^{\prime} \in L^{2}(\xi)\right)$ is meaningful. Let $\eta$ denote the standard Gaussian measure on $\mathbf{R}$. Then $\xi+\eta$ is a regular Borel measure on $\mathbf{R}$, hence there is a sequence $\left\{\varphi_{n}\right\}$ in $C_{0}(\mathbf{R})$ such that $\varphi_{n} \rightarrow F^{\prime}$ in $L^{2}(\xi+\eta)$; see $\left[\mathbf{1 2}\right.$; Proposition 7.9]. Define $F_{n}(t):=\int_{0}^{t} \varphi_{n}(s) d s$ for $t \in \mathbf{R}$. Evidently, $F_{n}$ is globally Lipschitz continuous, so that $F_{n} \circ u \in \mathcal{D}$ by the fundamental contraction property of Dirichlet spaces. Moreover, because $F_{n} \in C^{1}(\mathbf{R})$ we can apply [13; Theorem 3.2.2] to deduce that

$$
d \mu_{\left\langle F_{n} \circ u\right\rangle}=\left[\varphi_{n} \circ \tilde{u}\right]^{2} d \mu_{\langle u\rangle}
$$


for all $n \in \mathbf{N}$. Consequently,

$$
\mathcal{E}\left(F_{m} \circ u-F_{n} \circ u\right)=\frac{1}{2} \int_{E}\left[\varphi_{m} \circ \tilde{u}-\varphi_{n} \circ \tilde{u}\right]^{2} d \mu_{\langle u\rangle}=\int_{\mathbf{R}}\left[\varphi_{m}-\varphi_{n}\right]^{2} d \xi .
$$

It follows from (1.18) and the choice of $\left\{\varphi_{n}\right\}$ that the sequence $\left\{F_{n} \circ u\right\}$ is $\mathcal{E}$-Cauchy in $\mathcal{D}$. Also,

$$
\begin{aligned}
\left|F_{n}(t)-F(t)\right| & \leq \int_{0}^{|t|}\left|\varphi_{n}(s)-F^{\prime}(s)\right| d s \\
& \leq \sqrt{2 \pi} e^{t^{2} / 2} \int_{0}^{|t|}\left|\varphi_{n}(s)-F^{\prime}(s)\right|(2 \pi)^{-1 / 2} e^{-s^{2} / 2} d s \\
& \leq \sqrt{2 \pi} e^{t^{2} / 2}\left\|\varphi_{n}-F^{\prime}\right\|_{L^{2}(\eta)},
\end{aligned}
$$

from which it follows that $\lim _{n} F_{n}(t)=F(t)$ for each $t \in \mathbf{R}$. In particular, $F_{n} \circ u$ converges pointwise to $F \circ u$ as $n \rightarrow \infty$. Thus, $F \circ u$ is an element of the extended Dirichlet space $\mathcal{D}_{e}$; see $[\mathbf{1 3} ;$ page 35$]$. Since $F \circ u \in L^{2}(m)$ by hypothesis, we even have $F \circ u \in \mathcal{D}$ by $[\mathbf{1 3}$; Theorem 1.5.2 (iii)].

Because $\mathcal{E}\left(F_{n} \circ u-F \circ u\right) \rightarrow 0$ as $n \rightarrow \infty$, the left side of (1.17) converges in total variation norm to $d \mu_{\langle F \circ u\rangle}$; see $[\mathbf{1 3} ; 5.2 .12]$ and $[\mathbf{9} ;$ Lemma 5.6]. On the other hand, the right side of (1.17) converges (setwise) to $\left[F^{\prime} \circ \tilde{u}\right]^{2} d \mu_{\langle u\rangle}$, because $\varphi_{n} \circ \tilde{u} \rightarrow F^{\prime} \circ \tilde{u}$ in $L^{2}\left(\mu_{\langle u\rangle}\right)$. This proves (1.16).

\section{Theorem and Proof}

This section is devoted to the proof of the following theorem, our main result. By essentially unbounded we shall mean that $m(x \in E ;|u(x)|>t)>0$ for each $t>0$. If $\mathcal{D}$ contains no essentially unbounded elements, then $\mathcal{D} \subset L^{\infty}(m)$, in which case it is easy to see that every locally Lipschitz function operates on $\mathcal{D}$. A Borel set $D \subset E$ is said to be an exit set provided $\sup _{x \in E} \mathbf{P}^{x}[\tau(D)]<\infty$.

(2.1) Theorem. Let $K: \mathbf{R} \rightarrow \mathbf{R}$ be Borel measurable, with $K(0)=0$.

(a) If

$$
K \circ u \in \mathcal{D} \quad \text { for all } u \in \mathcal{D} \cap L^{\infty}(m),
$$

then $K$ is locally Lipschitz continuous, in the sense that for each $N \in \mathbf{N}$ there exists $\left.M_{N} \in\right] 0, \infty[$ such that

$$
|K(t)-K(s)| \leq M_{N}|t-s| \quad \text { for all } s, t \in[-N, N]
$$


Equivalently, $K$ is absolutely continuous, and the a.e. defined derivative $K^{\prime}$ is an element of $L_{\mathrm{loc}}^{\infty}(\mathbf{R})$.

(b) If

$$
K \circ u \in \mathcal{D} \quad \text { for all } u \in \mathcal{D}
$$

and if $\mathcal{D}$ contains an essentially unbounded element vanishing outside an exit set, then $K$ is globally Lipschitz continuous in the sense that there exists $M \in] 0, \infty[$ such that

$$
|K(t)-K(s)| \leq M|t-s| \quad \text { for all } s, t \in \mathbf{R}
$$

Equivalently, $K$ is absolutely continuous and the a.e. defined derivative $K^{\prime}$ is an element of $L^{\infty}(\mathbf{R})$.

(2.6) Remark. By an argument found on pp. 503-504 of [11], it can be shown that if there is a non-empty exceptional set (for example, if $X$ is Brownian motion in a Euclidean domain of dimension $d \geq 2$ ), then there is an essentially unbounded element of $\mathcal{D}$ vanishing outside an exit set. If there are no non-empty exceptional sets, then the transition measures of $X$ are absolutely continuous with respect to $m$ and each point of $E$ is regular for itself. The author does not know whether, in this latter case, the existence of an unbounded element of $\mathcal{D}$ guarantees the existence of such an element vanishing outside an exit set. An illustrative example of a process with no non-empty exceptional sets but with unbounded elements in its Dirichlet space is the 3 -dimensional Bessel process on $] 0, \infty[$.

Condenser potentials play a key role in the proof of Theorem (2.1), and in preparation we discuss some of their basic properties. Recall from [13; page 68] that a Borel set $B \subset E$ is quasi closed if (and only if) there is a nest $\left\{F_{n}\right\}$ of closed subsets of $E$ such that $B \cap F_{n}$ is a closed set for each $n \in \mathbf{N}$. For example, if $u \in \mathcal{D}$ and $\alpha \in \mathbf{R}$, then $\{x \in E ; \tilde{u}(x) \leq \alpha\}$ is quasi closed. Let $A$ and $B$ be quasi closed Borel subsets of $E$ such that $A \cap B$ is exceptional. The condenser potential

$$
p(x)=p_{A, B}(x):=\mathbf{P}^{x}\left[T_{A}<T_{B}\right]
$$

is an excessive function of $X$ killed at time $T_{B}$; consequently $p$ is quasi-continuous. Notice that $p(x)=1$ for q.e. $x \in A$ and $p(x)=0$ for q.e. $x \in B$. If the convex set

$$
\mathcal{U}=\mathcal{U}_{A, B}:=\{u \in \mathcal{D} ; \tilde{u}=1 \text { q.e. on } A, \tilde{u}=0 \text { q.e. on } B\}
$$


is nonempty, then

$$
\mathcal{E}(p)=\min \{\mathcal{E}(u) ; u \in \mathcal{U}\} .
$$

[Unless the process $X$ killed at $T_{B^{c}}$ is transient, there may be other elements of $\mathcal{U}$ at which the above minimum is attained.] Evidently, $\mathcal{U}$ is non-empty if and only if $p \in \mathcal{D}$. See [8] and $[\mathbf{2 0} ; \S 2]$ for more details on these matters.

If $p=p_{A, B} \in \mathcal{D}$, then there is a finite signed smooth measure $\nu=\nu_{A, B}$ such that

$$
\mathcal{E}(u, p)=\int_{E} \tilde{u} d \nu, \quad u \in \mathcal{D} .
$$

Indeed, the Hahn-Jordan decomposition $\nu^{+}-\nu^{-}$of $\nu$ is such that both $\nu^{+}$and $\nu^{-}$are smooth measures, and $\nu^{+}$is carried by $A$ while $\nu^{-}$is carried by $B$. (See the discussion on pp. $75-76$ of $[\mathbf{6}]$, and (2.15) below). Notice that

$$
C(A, B):=\mathcal{E}(p, p)=\int_{E} p d \nu=\nu(A)=\nu^{+}(E) .
$$

The following instance of the Bouleau-Hirsch "energy image density" theorem $[4$; Theorem I.7.1.1] is our main tool. This formula was suggested by a calculation in [14]. In the context of Brownian motion, a different proof can be fashioned out of the co-area and divergence formulas, as was shown to the author by Bruce Driver.

(2.9) Proposition. Fix $A$ and $B$ as above, and assume that $p=p_{A, B}$ lies in $\mathcal{D}$. Then for every Borel function $g:[0,1] \rightarrow[0, \infty]$,

$$
\frac{1}{2} \int_{E} g(p(x)) d \mu_{\langle p\rangle}(d x)=C(A, B) \int_{0}^{1} g(t) d t .
$$

Proof. It suffices to prove (2.10) when $g$ is continuous and real-valued. Define $G(y):=$ $\int_{0}^{y} g(t) d t$. Then $G \circ p \in \mathcal{D}$ and, by (2.7) and (2.8),

$$
\mathcal{E}(G \circ p, p)=\int_{A} G \circ p d \nu=G(1) \nu(A)=\int_{0}^{1} g(t) d t \cdot C(A, B) .
$$

On the other hand, by the polarized form of (1.16),

$$
\mathcal{E}(G \circ p, p)=\frac{1}{2} \int_{E} \mu_{\langle G \circ p, p\rangle}(d x)=\frac{1}{2} \int_{E} g(p(x)) \mu_{\langle p\rangle}(d x) .
$$

The proof of Theorem (2.1) will be accomplished through a series of lemmas. In the remainder of this section we suppose that $K: \mathbf{R} \rightarrow \mathbf{R}$ is a Borel function with $K(0)=0$, and that (2.2) holds. 
(2.11) Lemma. $K$ is continuous.

Proof. Arguing by contradiction, let us suppose that $K$ is not continuous. We may assume that $\liminf _{s \uparrow 1} K(s)<K(1)$; all other cases can be reduced to this case by suitable linear transformations of $K$. Thus, $\kappa:=\liminf _{t \uparrow 1} K(t)<K(1)$, so that there is a strictly increasing sequence $\left\{t_{n}\right\}$ of real numbers with $t_{0}=0$ and $\lim _{n} t_{n}=1$, such that $\lim _{n} K\left(t_{n}\right)=\kappa$. We can (and do) assume that $\sum_{n} \sqrt{t_{n}-t_{n-1}}<\infty$. (This can always be arranged by passing to a subsequence; let $n(k)$ be the smallest positive integer $n$ such that $t_{n} \geq 1-2^{-k}$, then $t_{n(k)}-t_{n(k-1)} \leq 2^{-(k-1)}$.) Define a second increasing sequence of positive numbers $\left\{\beta_{n}\right\}$ by their differences: $\beta_{0}:=0$ and

$$
\beta_{2 n}-\beta_{2 n-1}=\beta_{2 n+1}-\beta_{2 n}=C \sqrt{t_{n}-t_{n-1}}
$$

where the constant $C$ is chosen so that $\lim _{n} \beta_{n}=1$. Now let $w$ be a non-negative element of $\mathcal{D} \cap C_{0}(E)$ such that $\mathbf{P}^{m}\left[w\left(X_{0}\right) \leq \alpha, T_{\{w>1\}}<\zeta\right]>0$ for some $\left.\alpha \in\right] 0,1[$. Since $m(x \in$ $E ; w(x)=s\}$ is non-zero for at most countably many values of $s>0$, we can adjust $\alpha$ and $w$ slightly (by multiplying both by a suitable constant) to ensure that $m(x \in E ; w(x)=$ $1\}=0$ as well. Define relatively compact open sets $B_{1}, B_{2}, \ldots$ by $B_{n}:=\left\{w>\beta_{n}\right\}$. Let $p_{n} \in \mathcal{D}$ be the condenser potential defined by $p_{n}(x):=\mathbf{P}^{x}\left[T_{\overline{B_{2 n}}}<T_{B_{2 n-1}^{c}}\right], n=1,2, \ldots$. Then $p_{n}=1$ q.e. on $\overline{B_{2 n}}$ and $p_{n}=0$ q.e. on $B_{2 n-1}^{c}$. Define $u(x):=\sum_{n=1}^{\infty}\left(t_{n}-t_{n-1}\right) p_{n}(x)$. Each $p_{n}$ is quasi-continuous, and the series defining $u$ converges uniformly, and hence $u$ is quasi-continuous. Now $p_{n}$ is an element of $\mathcal{U}_{\overline{B_{2 n}}, B_{2 n-1}^{c}}:=\left\{v \in \mathcal{D} ; \tilde{v}=1\right.$ q.e. on $\overline{B_{2 n}}, \tilde{v}=0$ q.e. on $\left.B_{2 n-1}^{c}\right\}$ of least energy; thus, if we set

$$
w_{n}:=\left[\left(\beta_{2 n}-\beta_{2 n-1}\right)^{-1}\left(w-\beta_{2 n-1}\right)^{+}\right] \wedge 1 \in \mathcal{U}_{\overline{B_{2 n}}, B_{2 n-1}^{c}},
$$

then

$$
\mathcal{E}\left(p_{n}\right) \leq \mathcal{E}\left(w_{n}\right) \leq\left(\beta_{2 n}-\beta_{2 n-1}\right)^{-2} \mathcal{E}(w)
$$

In view of $(2.7)$ and the subsequent discussion, $\mathcal{E}\left(p_{j}, p_{k}\right)=0$ if $j \neq k$. Therefore, writing 
$u_{n}=\sum_{k=1}^{n}\left(t_{k}-t_{k-1}\right) p_{k}$, we have

$$
\begin{aligned}
\mathcal{E}\left(u_{n}\right) & =\sum_{k=1}^{n}\left(t_{k}-t_{k-1}\right)^{2} \mathcal{E}\left(p_{k}\right) \\
& \leq \mathcal{E}(w) \sum_{k=1}^{n}\left(t_{k}-t_{k-1}\right)^{2}\left(\beta_{2 k}-\beta_{2 k-1}\right)^{-2} \\
& =\mathcal{E}(w) \cdot C^{-2} \sum_{k=1}^{n}\left(t_{k}-t_{k-1}\right) \leq \mathcal{E}(w) \cdot C^{-2}
\end{aligned}
$$

In addition, because $u_{n}$ vanishes q.e. outside $B_{1}$ and $0 \leq u_{n} \leq 1$, we have $\left\|u_{n}\right\|_{2}^{2} \leq$ $m\left(B_{1}\right) \leq \beta_{1}^{-1} \int_{E} w d m<\infty$. By the Banach-Saks theorem, the Cesàro means of some subsequence of $\left\{u_{n}\right\}$ converge in $\mathcal{D}$; the limit must coincide with the pointwise limit of the $\left\{u_{n}\right\}$, namely $u$. It follows that $u \in \mathcal{D}$. Notice that $0 \leq u \leq 1, u \equiv 1$ on $\{w>1\}$, and $u=t_{n}$ q.e. on $\overline{B_{2 n}} \backslash B_{2 n+1} \supset\left\{x \in E ; \beta_{2 n}<w(x)<\beta_{2 n+1}\right\}$.

If $h: \mathbf{R} \rightarrow \mathbf{R}$ is Borel measurable, then

$$
\operatorname{ess} \lim \sup h(s):=\lim _{\delta \downarrow} \operatorname{ess}_{s \in] t, t+\delta[} h(s)
$$

where "ess sup" denotes essential supremum with respect to Lebesgue measure. The notions ess liminf $\operatorname{s\downarrow t}_{t}$ and ess $\lim _{s \downarrow t}$ are defined analogously, as are their counterparts from the left. If $v \in \mathcal{D}$ then $t \mapsto \tilde{v}\left(X_{t}(\omega)\right)$ is continuous on $\left[0, \zeta(\omega)\right.$ [ for $\mathbf{P}^{m}$-a.e. $\omega \in \Omega$. Also, $\mathbf{P}^{m}\left[\tilde{v}\left(X_{t}\right) \neq v\left(X_{t}\right)\right]=m P_{t}(\tilde{v} \neq v) \leq m(\tilde{v} \neq v)=0$. Consequently, by Fubini's theorem, $\tilde{v}\left(X_{t}(\omega)\right)=v\left(X_{t}(\omega)\right)$ for a.e. $t \geq 0$, for $\mathbf{P}^{m}$-a.e. $\omega \in \Omega$. Therefore,

$$
\left.\tilde{v}\left(X_{t}(\omega)\right)=\underset{s \uparrow t}{\operatorname{ess} \lim } v\left(X_{s}(\omega)\right)=\underset{s \downarrow t}{\operatorname{ess} \lim } v\left(X_{s}(\omega)\right), \quad \text { for all } t \in\right] 0, \zeta(\omega)[,
$$

for $\mathbf{P}^{m}$-a.e. $\omega \in \Omega$. Define $\mathbf{P}^{*}[\cdot]:=\int_{\{w \leq \alpha\}} \mathbf{P}^{x}[\cdot] m(d x)$, and let $\tau$ denote the hitting time of $\{w>1\}$. Observe that

$$
\mathbf{P}^{m}\left[\int_{0}^{\infty} 1_{\{w=1\}}\left(X_{t}\right) d t\right]=\int_{0}^{\infty} m P_{t}(w=1) d t \leq \int_{0}^{\infty} m(w=1) d t=0 .
$$

This implies that

$$
\left.\left.\mathbf{P}^{*}\left[\exists \delta>0 \text { such that } w\left(X_{t}\right)=1 \text { for all } t \in\right] \tau-\delta, \tau\right] ; \tau<\zeta\right]=0
$$

Recall that $w$ and $\alpha$ were chosen so that $\mathbf{P}^{*}[\tau<\zeta]>0$, and clearly $\mathbf{P}^{*}[\tau=0]=0$. Let $\omega \in\left\{X_{0} \leq \alpha\right\}$ be a sample point such that $t \mapsto X_{t}(\omega)$ is continuous and (2.12) holds 
with $v=K \circ u$. Because of (2.13) we may suppose that $t \mapsto w\left(X_{t}(\omega)\right)$ is not identically 1 in any interval of the form $] \tau(\omega)-\delta, \tau(\omega)[$. Thus, given $\delta \in] 0, \tau(\omega)\left[\right.$, there exists $t_{\delta} \in$ ]$\tau(\omega)-\delta, \tau(\omega)$ [ such that $w\left(X_{t_{\delta}}(\omega)\right)<1$, which implies that $t \mapsto w\left(X_{t}(\omega)\right)$ takes on every value between $w\left(X_{t_{\delta}}(\omega)\right)$ and 1 during the time interval $] \tau(\omega)-\delta, \tau(\omega)$ [. Consequently, there is a positive integer $n_{0}(\omega) \geq \delta^{-1}$ such that for each $n \geq n_{0}(\omega), X_{t}(\omega) \in\left\{\beta_{2 n}<\right.$ $\left.w<\beta_{2 n+1}\right\} \subset \bar{B}_{2 n} \backslash B_{2 n+1}$ for some $\left.t \in\right] \tau(\omega)-\delta, \tau(\omega)\left[\right.$. Because $t \mapsto X_{t}(\omega)$ is continuous and $\left\{\beta_{2 n}<w<\beta_{2 n+1}\right\}$ is open, we conclude that the Lebesgue measure of $\{t \in] \tau(\omega)-$ $\delta, \tau(\omega)\left[; X_{t}(\omega) \in \bar{B}_{2 n} \backslash B_{2 n+1}\right\}$ is strictly positive for each $n \geq n_{0}(\omega)$. Because $u(x)=t_{n}$ for q.e. $x \in \bar{B}_{2 n} \backslash B_{2 n+1}$ we therefore have

$$
\operatorname{essinf}_{\tau(\omega)-\delta<t<\tau(\omega)} K\left(u\left(X_{t}(\omega)\right)\right) \leq \inf _{n \geq n_{0}(\omega)} K\left(t_{n}\right) .
$$

In view of (2.12) (which, in particular, guarantees the existence of ess $\lim _{t \uparrow \tau} K\left(u\left(X_{t}\right)\right)$ ), this last display implies that

$$
\widetilde{K \circ u}\left(X_{\tau}\right)=\underset{t \uparrow \tau}{\operatorname{ess}} \lim K\left(u\left(X_{t}\right)\right) \leq \lim _{n} K\left(t_{n}\right)=\kappa<K(1)
$$

$\mathbf{P}^{*}$-a.s. on the event $\{\tau<\zeta\}$. On the other hand, because $\{w>1\}$ is open, if $\tau(\omega)<\zeta(\omega)$ then for every $\delta>0$ the set $\{t \in] \tau(\omega), \tau(\omega)+\delta\left[; w\left(X_{t}(\omega)\right)>1\right\}$ has positive Lebesgue measure. Because $u \equiv 1$ on $\{w>1\}$ we therefore have

$$
\widetilde{K \circ u}\left(X_{\tau}\right)=\underset{t \downarrow \tau}{\operatorname{ess} \lim } K\left(u\left(X_{t}\right)\right)=K(1)
$$

$\mathbf{P}^{*}$-a.s. on the event $\{\tau<\zeta\}$. Since $\mathbf{P}^{*}[\tau<\zeta]>0$, the last two displays provide the desired contradiction.

(2.14) Lemma. $K$ is absolutely continuous, and $K^{\prime} \in L_{\mathrm{loc}}^{2}(\mathbf{R})$.

Proof. We begin by showing that $K$ is absolutely continuous on ]0, 1[ with $K^{\prime} \in L_{\text {loc }}^{2}(] 0,1[)$. For this it suffices to prove that there is a constant $C$ such that

$$
\left|\int_{0}^{1} K(s) \psi^{\prime}(s) d s\right| \leq C\|\psi\|_{2}, \quad \text { for all } \psi \in C_{0}^{\infty}(] 0,1[)
$$

Fix $\psi \in C_{0}^{\infty}(] 0,1[)$ and define $\Psi(t):=\int_{0}^{t} \psi(s) d s$. Let $U$ and $V$ be non-empty open relatively compact subsets of $E$ such that $\bar{U} \subset V$. Let $p$ be the condenser potential 
associated with $A=\bar{U}$ and $B=V^{c}$. From $[\mathbf{6} ;(3.4)]$ we know that there are PCAFs $C^{A}$ and $C^{B}$ with $C^{A}$ (resp. $C^{B}$ ) increasing only when $X$ is in $A$ (resp. in $B$ ) such that the Fukushima decomposition associated with $p$ is

$$
p\left(X_{t}\right)-p\left(X_{0}\right)=M_{t}^{p}+N_{t}^{p}=M_{t}^{p}-C_{t}^{A}+C_{t}^{B}, \quad 0 \leq t<\zeta .
$$

Indeed, $C^{A}$ (resp. $C^{B}$ ) is the PCAF with Revuz measure [13; Section 5.1] $\nu^{+}$(resp. $\nu^{-}$), where $\nu^{+}-\nu^{-}$is the Hahn-Jordan decomposition of the signed smooth measure associated with $p$. Now $\Psi \circ p \in \mathcal{D}$, and from (2.16) and Itô's formula it follows that

$$
N_{t}^{\Psi \circ p}=\frac{1}{2} \int_{0}^{t} \psi^{\prime}\left(p\left(X_{s}\right)\right) d\left\langle M^{p}\right\rangle_{s}, \quad t \geq 0 .
$$

By a result of Nakao [19; $§ 3],(2.17)$ yields

$$
\mathcal{E}(v, \Psi \circ p)=-\frac{1}{2} \int_{E} \tilde{v}(x) \psi^{\prime}(p(x)) \mu_{\langle p\rangle}(d x),
$$

for any bounded $v \in \mathcal{D}$. In particular, by $(2.10)$,

$$
\mathcal{E}(K \circ p, \Psi \circ p)=-\frac{1}{2} \int_{E} K(p(x)) \psi^{\prime}(p(x)) \mu_{\langle p\rangle}(d x)=-C(A, B) \int_{0}^{1} K(s) \psi^{\prime}(s) d s .
$$

On the other hand,

$$
\begin{aligned}
|\mathcal{E}(K \circ p, \Psi \circ p)| & \leq \mathcal{E}(K \circ p)^{1 / 2} \mathcal{E}(\Psi \circ p)^{1 / 2} \\
& =\mathcal{E}(K \circ p)^{1 / 2} C(A, B)^{1 / 2}\|\psi\|_{2},
\end{aligned}
$$

where we have used (1.14) and (2.10) for the equality. Thus (2.15) holds with $C=$ $[\mathcal{E}(K \circ p) / C(A, B)]^{1 / 2}$.

Now notice that $J(x):=K(|4 x-1|-1)$ satisfies (2.2). By the preceding paragraph, $J$ is absolutely continuous on $] 0,1\left[\right.$ and $J^{\prime} \in L_{\text {loc }}^{2}(] 0,1[)$. But the restriction of $J$ to $[1 / 4,3 / 4]$ is $x \mapsto K(4 x-2)$. It follows that $K$ is absolutely continuous on $[-1,1]$ and that $K^{\prime} \in L^{2}[-1,1]$. Finally, for $b>0$, the preceding applies to $K_{b}(x):=K(b x)$, the upshot being that $K$ is absolutely continuous on $[-b, b]$ with $K^{\prime} \in L^{2}[-b, b]$.

(2.19) Lemma. $K^{\prime} \in L_{\mathrm{loc}}^{\infty}(\mathbf{R})$.

Proof. Arguing as at the end of the proof of (2.14), it is enough to show that $K^{\prime} \in L^{\infty}[0,1]$. For this we need only to show that if $0 \leq f \in L^{1}[0,1]$, then

$$
\int_{0}^{1}\left[K^{\prime}(s)\right]^{2} f(s) d s<\infty .
$$


Fix such an $f$ and define $\varphi:[0,1] \rightarrow[0,1]$ by the formula

$$
t=\gamma^{-1} \int_{0}^{\varphi(t)} \frac{1}{1+f(s)} d s, \quad 0 \leq t \leq 1
$$

where $\gamma:=\int_{0}^{1}[1+f(s)]^{-1} d s$. Evidently, $\varphi$ is a strictly increasing map of $[0,1]$ onto itself, and $\varphi$ is an absolutely continuous function with $\varphi(0)=0$ and

$$
\varphi^{\prime}(t)=\gamma[1+f(\varphi(t))]
$$

for Lebesgue a.e. $t \in[0,1]$. Let the condenser potential $p$ be as in the proof of Lemma (2.14). Observe that $\varphi \circ p$ is bounded and $m(\varphi \circ p \neq 0) \leq m(p>0)<\infty$; therefore $\varphi \circ p \in L^{2}(m)$. By Proposition (1.15), $\varphi \circ p \in \mathcal{D}$ because

$$
\frac{1}{2} \int_{E}\left[\varphi^{\prime} \circ p\right]^{2} d \mu_{\langle p\rangle}=C(A, B) \int_{0}^{1}\left[\varphi^{\prime}(s)\right]^{2} d s=\gamma C(A, B) \int_{0}^{1}[1+f(t)] d t<\infty .
$$

Therefore $K \circ \varphi \circ p \in \mathcal{D}$, and hence

$$
\begin{aligned}
\int_{0}^{1}\left[K^{\prime}(t)\right]^{2}[1+f(t)] d t & =\frac{1}{\gamma} \int_{0}^{1}\left[K^{\prime}(\varphi(s)]^{2}\left[\varphi^{\prime}(s)\right]^{2} d s\right. \\
& =\frac{1}{2 \gamma C(A, B)} \int_{E}\left[K^{\prime}(\varphi(p(x)))\right]^{2}\left[\varphi^{\prime}(p(x))\right]^{2} \mu_{\langle p\rangle}(d x) \\
& =\frac{1}{\gamma C(A, B)} \mathcal{E}(K \circ \varphi \circ p)<\infty .
\end{aligned}
$$

(2.21) Lemma. If $\mathcal{D}$ contains an essentially unbounded element vanishing outside an exit set, then $K^{\prime} \in L^{\infty}(\mathbf{R})$.

Proof. It suffices to show that $K^{\prime} \in L^{\infty}[0, \infty[$, and for this it is enough to show that

$$
\int_{0}^{\infty}\left[K^{\prime}(s)\right]^{2} f(s) d s<\infty
$$

for each non-negative $f \in L^{1}[0, \infty[$. Fix such an $f$. Let $v$ be an essentially unbounded element of $\mathcal{D}$ such that $\{\tilde{v} \neq 0\}$ is an exit set; substituting $|v|$ for $v$ if necessary, we can and do assume that $v \geq 0$. For $n \in \mathbf{N}$ define quasi closed sets $A_{n}:=\{\tilde{v} \geq 2 n+1\}$ and $B_{n}:=\{\tilde{v} \leq 2 n\}$, and let $p_{n}$ be the associated condenser potential $p_{A_{n}, B_{n}}$. Because $v$ is essentially unbounded, the condenser capacities

$$
C_{n}:=C\left(A_{n}, B_{n}\right)
$$


are strictly positive and finite. Notice that

$$
\mathcal{E}\left(p_{n}, p_{m}\right)=0 \quad \text { if } m \neq n
$$

Because

$$
C_{n} \leq \mathcal{E}\left((v-2 n)^{+} \wedge 1\right)=\frac{1}{2} \int_{E} 1_{[2 n, 2 n+1]}(\tilde{v}(x)) \mu_{\langle v\rangle}(d x) \rightarrow 0 \quad \text { as } n \rightarrow \infty
$$

we have $\sum_{n} C_{n}^{-1}=\infty$. Next observe that

$$
x^{2} \leq \int_{0}^{x}\left[e^{-s}+f(s)\right] d s \cdot \int_{0}^{x}\left[e^{-s}+f(s)\right]^{-1} d s
$$

and hence the integrability of $s \mapsto e^{-s}+f(s)$ implies that

$$
\lim _{x \rightarrow \infty} \int_{0}^{x}\left[e^{-s}+f(s)\right]^{-1} d s=\infty
$$

Therefore a strictly increasing function $\varphi:[0, \infty[\rightarrow[0, \infty[$ is well-defined by

$$
t=\int_{0}^{\varphi(t)} \frac{1}{e^{-s}+f(s)} d s, \quad t \geq 0
$$

Clearly, $\varphi^{\prime}(s)=e^{-\varphi(s)}+f(\varphi(s))$ for Lebesgue a.e. $s>0, \varphi(0)=0$, and $\lim _{s \rightarrow \infty} \varphi(s)=\infty$.

Let us now check that the function $u$ defined by

$$
u(x):=\varphi\left(\sum_{k=1}^{\infty} C_{k}^{-1} p_{k}(x)\right), \quad x \in E
$$

is an element of $\mathcal{D}$. Proposition (1.15) implies that for each $n \in \mathbf{N}$,

$$
u_{n}(x):=\varphi\left(\sum_{k=1}^{n} C_{k}^{-1} p_{k}(x)\right), \quad x \in E,
$$

is an element of $\mathcal{D}$. Clearly, $u$ is the pointwise limit of the sequence $\left\{u_{n}\right\}$. An application of the Banach-Saks theorem (as in the proof of Lemma (2.11)) shows that the asserted inclusion of $u \in \mathcal{D}$ will follow once we check that both $\mathcal{E}\left(u_{n}\right)$ and $\left\|u_{n}\right\|_{2}$ are bounded functions of $n$. Now, because $p_{k}$ is 1 on the support of $p_{j}$ for $j>k$, we have $\mu_{\left\langle p_{j}, p_{k}\right\rangle}=0$ 
for $j>k$. Thus, defining $x_{0}:=0$ and $x_{k}:=C_{1}^{-1}+\cdots C_{k}^{-1}$ for $k \geq 1$, the key formula (2.10) yields that

$$
\begin{aligned}
\mathcal{E}\left(u_{n}\right) & =\sum_{k=1}^{n} \int_{E}\left[\varphi^{\prime}\left(x_{k-1}+C_{k}^{-1} p_{k}(x)\right)\right]^{2} C_{k}^{-2} \mu_{\left\langle p_{k}\right\rangle}(d x) \\
& =\sum_{k=1}^{n} C_{k} \int_{0}^{1}\left[\varphi^{\prime}\left(x_{k-1}+C_{k}^{-1} s\right)\right]^{2} C_{k}^{-2} d s \\
& =\sum_{k=1}^{n} \int_{0}^{1}\left[\varphi^{\prime}\left(x_{k-1}+C_{k}^{-1} s\right)\right]^{2} C_{k}^{-1} d s \\
& =\sum_{k=1}^{n} \int_{0}^{C_{k}^{-1}}\left[\varphi^{\prime}\left(x_{k-1}+t\right)\right]^{2} d t \\
& =\int_{0}^{x_{n}}\left[\varphi^{\prime}(z)\right]^{2} d z \leq \int_{0}^{\infty}\left[\varphi^{\prime}(z)\right]^{2} d z \\
& =\int_{0}^{\infty} \varphi^{\prime}\left(\varphi^{-1}(t)\right) d t \\
& =\int_{0}^{\infty}\left[e^{-t}+f(t)\right] d t<\infty .
\end{aligned}
$$

Because $u_{n}$ vanishes outside the exit set $\{\tilde{v}>0\}$, we have (by [22; Theorem 3.1] or [10; (1.19)] applied to $X$ killed at the exit time from $\{\tilde{v}>0\}$ )

$$
\left\|u_{n}\right\|_{2}^{2} \leq c_{1} \mathcal{E}\left(u_{n}\right) \leq c_{1} \int_{0}^{\infty}\left[e^{-t}+f(t)\right] d t<\infty
$$

where $c_{1}:=\sup _{x \in E} \mathbf{P}^{x}\left[T_{\{\tilde{v}=0\}}\right]<\infty$. Thus, $u \in \mathcal{D}$, hence $K \circ u \in \mathcal{D}$. Computing as above, we find, because $\lim _{n \rightarrow \infty} x_{n}=\infty$,

$$
\infty>\mathcal{E}(K \circ u)=\int_{0}^{\infty}\left[K^{\prime}(t)\right]^{2}\left[e^{-t}+f(t)\right] d t \geq \int_{0}^{\infty}\left[K^{\prime}(t)\right]^{2} f(t) d t
$$

as desired.

Acknowledgment. I thank the referee, whose careful reading of the manuscript led to the elimination of several errors.

\section{References}

[1] A. Ancona, Continuité des contractions dans les espaces de Dirichlet, Séminaire de Théorie du Potentiel de Paris, no. 2, pp. 1-26. Lecture Notes in Math. 563 Springer, Berlin, 1976. [MR 58 \#28567]

[2] J. Appell and P. P. Zabrejko, Nonlinear Superposition Operators, Cambridge University Press, Cambridge, 1990. [MR 91k:47168] 
[3] R. M. Blumenthal and R. K. Getoor, Markov Processes and Potential Theory, Academic Press, New York-London, 1968 [MR 41 \#9348]

[4] N. Bouleau and F. Hirsch, Formes de Dirichlet générales et densité des variables aléatoires réelles sur l'espace de Wiener, J. Funct. Anal. 69 (1986), 229-259. [MR 88d:60196]

[5] G. Bourdaud, Le calcul fonctionnel dans les espaces de Sobolev, Invent. Math. 104 (1991), 435-446. [MR 93b:46053]

[6] Z.-Q. Chen, P. J. Fitzsimmons, and R. Song, Crossing estimates for symmetric Markov processes, Probab. Theory Related Fields 120 (2001), 68-84.

[7] Z.-Q. Chen, Z.-M. Ma, and M. Röckner, Quasi-homeomorphisms of Dirichlet forms, Nagoya Math. J. 136 (1994), 1-15. [MR 95m:31020]

[8] K. L. Chung and R. K. Getoor, The condenser problem, Ann. Probab. 5 (1977), 82-86. [MR 55 \#1501]

[9] P. J. Fitzsimmons, Absolute continuity of symmetric diffusions, Ann. Probab. 25 (1997), 230-258. [MR 98c:60097]

[10] P. J. Fitzsimmons, Hardy's inequality for Dirichlet forms, J. Math. Anal. Appl. 250 (2000), 548-560. [MR 2001h:31010]

[11] P. J. Fitzsimmons and R. K. Getoor, On the potential theory of symmetric Markov processes, Math. Ann. 281 (1988), 495-512. [MR 89k:60110]

[12] G. B. Folland, Real Analysis (Second edition), Wiley, New York, 1999. [MR 2000c:00001]

[13] M. Fukushima, Y. Ōshima, and M. Takeda, Dirichlet Forms and Symmetric Markov Processes, Walter de Gruyter \& Co., Berlin, 1994. [MR 96f:60126]

[14] M. Fukushima and J. Ying, A note on regular Dirichlet subspaces, Proc. Amer. Math. Soc., 131 (2003), 1607-1610. 2003.

[15] D. A. Labutin, Superposition operator in Sobolev spaces on domains, Proc. Amer. Math. Soc. 128 (2000), 3399-3403. [MR 2001b:47106]

[16] Z.-M. Ma, and M. Röckner, Introduction to the Theory of (Nonsymmetric) Dirichlet Forms, Springer-Verlag, Berlin, 1992. [MR 94d:60119]

[17] M. Marcus and V. J. Mizel, Complete characterization of functions which act, via superposition, on Sobolev spaces, Trans. Amer. Math. Soc. 251 (1979), 187-218. [MR 80j:46055]

[18] M. Marcus and V. J. Mizel, Every superposition operator mapping one Sobolev space into another is continuous, J. Funct. Anal. 33 (1979), 217-229. [MR 80h:47039]

[19] S. Nakao, Stochastic calculus for continuous additive functionals of zero energy, Z. Wahrsch. Verw. Gebiete 68 (1985), 557-578. [MR 86c:60082] 
[20] Y. Öshima and T. Yamada, On some representations of continuous additive functionals locally of zero energy, J. Math. Soc. Japan 36 (1984), 315-339. [MR 85j:60136]

[21] M. Sharpe, General Theory of Markov Processes, Academic Press, San Diego, 1988. [MR 89m:60169]

[22] P. Stollmann and J. Voigt, Perturbation of Dirichlet forms by measures, Potential Anal. 5 (1996), 109-138. [MR 97e:47065 ]

Department of Mathematics, 0112

University of California, San Diego

9500 Gilman Drive

La Jolla, CA 92093-0112 